\title{
A SIMPLE SOI-CMOS COMPATIBLE TECHNOLOGY TO MAKE FLEXIBLE ELECTRONICS \\ Hongen $\mathrm{Tu}^{1}$ and Yong $\mathrm{Xu} \mathrm{u}^{1}$ \\ ${ }^{1}$ Wayne State University, Detroit, MI, USA
}

\begin{abstract}
Flexible sensors or electronics are expected to play important roles in various healthcare and medical applications. Fabrication techniques like direct coating and patterning organic or inorganic semiconductor materials on flexible substrates are ideal to reduce the cost. However, high temperature processes are not allowed due to the flexible substrate, limiting the choice of materials and reduce the performance of the device. The recently developed transfer printing technology also has limitations due to its incompatibility with mainstream CMOS technology. This paper reports a simple SOI-CMOS compatible technology to make flexible electronics. This new technology takes advantage of $\mathrm{XeF}_{2}$ isotropic gas phase silicon etchant and conformal parylene $\mathrm{C}$ coating, making the process SOI-CMOS compatible.
\end{abstract}

\section{INTRODUCTION}

Flexible sensors or electronics are believed to have significant impacts on wearable health monitoring, medical implants and many other applications. Over the last two decades, many different approaches of making flexible sensors or electronics have been proposed and researched. The most straightforward one is to fabricate directly on flexible substrate. Actually, similar technology has existed for a long time in the electronics industry, for example, the widely used flexible printed circuitry technology and the thin film transistor (TFT) technology on flexible substrates. In reference [1], a flexible large area position sensitive detector was developed by depositing amorphous silicon on Kapton polyimide substrate. In reference [2], a flexible multichannel sieve electrode for interfacing regenerating peripheral nerves was fabricated on polyimide film by using a silicon wafer as a support for a much better dimension control. Simple MEMS structures on plastic substrates, such as amorphous silicon air-gap resonators, have been demonstrated as well [3]. The simple fabrication process and low cost are the main advantages of direct fabrication on flexible substrate. Large area flexible sensors or electronics can be fabricated in this way economically. However, since the process temperature is limited due to the flexible substrate, high temperature processes are ruled out and the material properties are not optimized. Moreover, due to this temperature limit, it will be almost impossible to integrate CMOS circuits and many MEMS transducers to the flexible substrate monolithically.

We have previously reported a flexible skin technology based on silicon island structure [4-6]. The basic structure of the flexible skin is arrays of silicon islands sandwiched by two layers of polymer. The biggest advantage of this technology is its compatibility with MEMS and CMOS since MEMS devices and CMOS circuits can be fabricated on the silicon wafer before the formation of the flexible skin. The minimum bending radius is determined by the size the of the silicon islands. Based on this technology, flexible shear stress sensor skins [4], the integration of CMOS with the flexible skin [5], and intelligent textiles [7] have been demonstrated.

Recently, Dr. John Rogers' group developed a flexible electronics technology using an innovative "transfer printing" method $[8,9]$. Namely, transistors and other devices are fabricated first on SOI wafers and then transferred to flexible substrates by "printing". Many exciting applications have been demonstrated [10, 11]. Nevertheless, the releasing step is generally incompatible with commercial CMOS process. Therefore, this process still cannot fully take advantage of the mainstream CMOS technology. In addition, the metal interconnection among discrete components was formed on the flexible substrate after transferring, limiting the device density and performance.

This paper reports a simple SOI-CMOS compatible technology which can be used to fabricate flexible electronics without the limitations of "printing" technique. The most unique part of our technology is the releasing step. All the electronic components of our device, including metal interconnects and discrete silicon islands, can be fabricated right before the final releasing step. The releasing step includes two parylene $\mathrm{C}$ film depositions. The first parylene layer is used as a mask to release the devices and a supporting layer for released devices. The second parylene layer functions as an encapsulation layer for the devices. $\mathrm{XeF}_{2}$ isotropic gas phase silicon etchant is used here in the releasing step to etch away the bulk silicon in the handle wafer. Parylene $\mathrm{C}$ is selected as the flexible substrate because parylene $\mathrm{C}$ deposition is a stress-free conformal process, and parylene $\mathrm{C}$ possesses excellent properties such as chemical inertness, biocompatibility, low leakage current and low gas permeability. We have previously utilized $\mathrm{XeF}_{2}$ isotropic etching and parylene conformal deposition to fabricate microchannels and individually-addressable microtubes [12, 13]. Here to demonstrate the concept of the new flexible electronics technology, silicon strain gauges were first fabricated.

\section{FABRICATION}

The fabrication process starts with a Soitec SOI wafer with a device layer of $0.36 \mathrm{um}$ and a buried oxide (BOX) layer of $0.5 \mathrm{um}$. As shown in Fig. 1 (a), phosphorus diffusion source was used to dope the Si device layer. Silicon thermal oxide was used as a diffusion mask here. The exposed device layer became highly doped. Then as shown in Fig. 1 (b), individual Si island was patterned and the exposed BOX layer were removed sequentially. $\mathrm{Cr} / \mathrm{Au}$ traces and contact pads were then sputtered and patterned as shown in Fig. 1 (c). Here we chose Au because of its excellent ductility. In the later development, we will use CMOS-compatible metals such as Al. It can be observed that the electronic components can be fabricated via standard CMOS process up to this point since there is no temperature limit of fabrication process.

Next, a few more steps were performed to transfer devices from Si substrate into flexible parylene C substrate. Firstly, a 3 um thick parylene $\mathrm{C}$ layer was deposited as illustrated in Fig. 1 (d). Then arrays of small windows (8um $\times 25 \mathrm{um}) 60 \mathrm{um}$ apart were opened in this parylene layer using $\mathrm{O}_{2}$ plasma (step (e)). Note that all the wide metal traces/pads and silicon islands were also etched selectively to open the windows at this step. Then, $\mathrm{XeF}_{2}$, an isotropic gas phase silicon etchant, was used to completely undercut the silicon underneath through the windows formed in the previous step (step (f)). Note that silicon islands were protected by both parylene $\mathrm{C}$ on the top and $\mathrm{BOX}$ on the bottom. At this point both strain gauges and metal traces/pads were supported by a free-standing perforated parylene $\mathrm{C}$ membrane. Next, a second parylene $C$ layer (10 um) was conformally deposited to encapsulate the strain gauges and metal traces/pads, and simultaneously seal the 
etching windows as shown in Fig. 1 (g). Oxygen plasma was then used to open bonding pads and shape the outline of the flexible device. Finally the flexible device can be simply peeled off from the Si substrate. Note that there were two physically separated parylene $\mathrm{C}$ layers after step (h). Depending on the application, we can choose to either keep or remove the scalloped bottom parylene $\mathrm{C}$ layer by just modifying the final releasing mask. Because these two parylene $\mathrm{C}$ layers formed an enclosed space, by keeping the bottom parylene layer, we could easily integrate functioning microchannels or microtubes in the system. We cut the bottom layer here to demonstrate a simple strain gauge embedded in top parylene $\mathrm{C}$ layer.

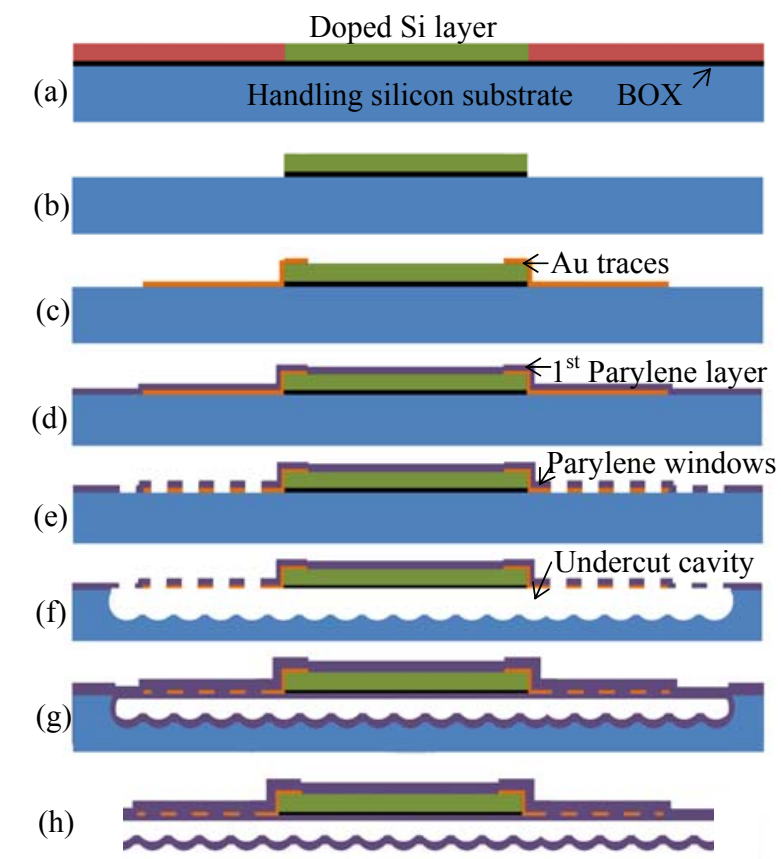

Figure 1: Simplified process flow: (a) Phosphorus diffusion.(b)Pattern the device layer and remove BOX layer.(c)Au deposition and patterning to form traces and pads. (d) $1^{\text {st }} 3 \mathrm{um}$ parylene deposition. (e)Pattern the parylene windows and etch away underneath metal traces.(f) $\mathrm{XeF}_{2}$ etching to release the devices. (g) $2^{\text {nd }} 10$ um parylene deposition. (h) Final released device.

\section{RESULTS AND DISCUSSION}

A fabricated flexible device is shown in Fig. 2, where the flexibility can be clearly observed. All the devices and metal traces/pads are held in position and protected by conformally coated transparent parylene $\mathrm{C}$ films. We have several strain gauges placed across the device which can be electrically accessed by $1 \mathrm{~mm} \times 1 \mathrm{~mm}$ large $\mathrm{Au}$ contact pads to facilitate later testing.

The magnified view of two perpendicular strain gauges can be found in Fig. 3. In this figure, both narrow metal traces and wide perforated metal traces can be observed. Wide metal traces will make our device more durable and they are also required for contact pads. Note that arrays of small dimples, which are resealed parylene $\mathrm{C}$ windows after second parylene $\mathrm{C}$ deposition, can be observed on the surface as shown in Fig. 3.

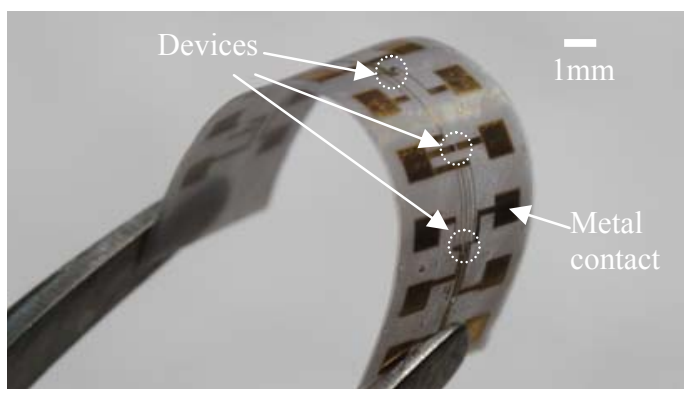

Figure 2: A bent flexible device held by a pair of tweezers.

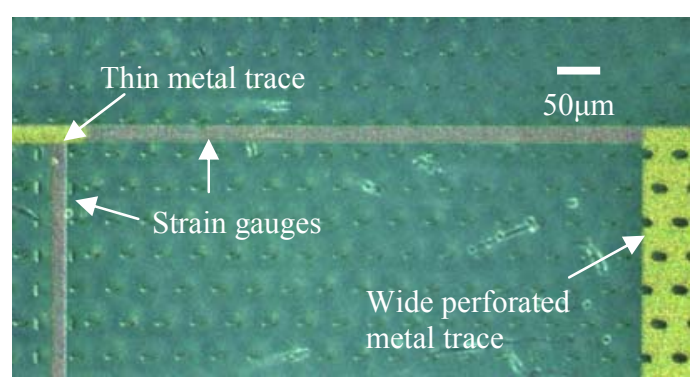

Figure 3: Optical micrograph of two perpendicular strain gauges.

It is also interesting to observe how the $\mathrm{XeF}_{2}$ etches the handle silicon substrate. Figure 4 shows the SEM image of the bottom parylene $\mathrm{C}$ layer after it was peeled off from the substrate. This layer of parylene $\mathrm{C}$ film was formed during the second deposition when the parylene $\mathrm{C}$ windows were sealed. The honey-comb shape reflects the silicon surface resulting from by the $\mathrm{XeF}_{2}$ isotropic etching.

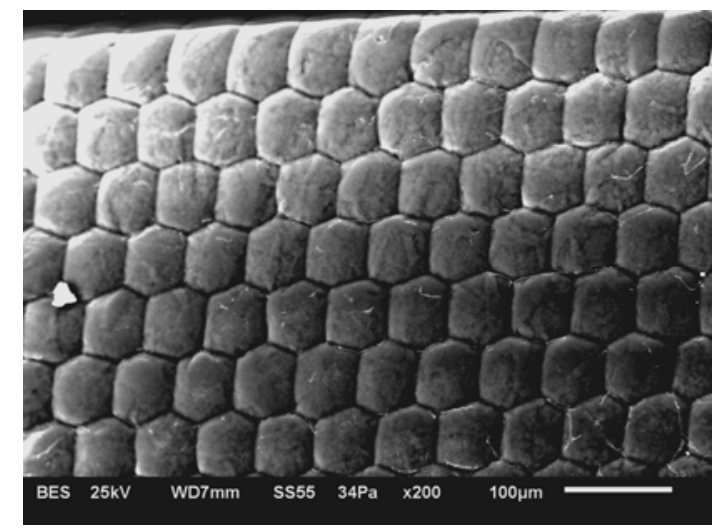

Figure 4: SEM image of the bottom honey-comb shaped parylene layer

More detailed information of sealed parylene windows is provided by Fig. 5. The original opening size of the perforated parylene $\mathrm{C}$ window was only $8 \mathrm{um}$ wide. The second $10 \mathrm{um}$ parylene $\mathrm{C}$ deposition was more than enough to completely seal the windows and provides enhanced strength to the film. The perforated contact metal pads can also be observed. Note that this SEM image was taken without sputtering a thin $\mathrm{Au}$ layer. Consequently, the exposed contact pad was very bright. 


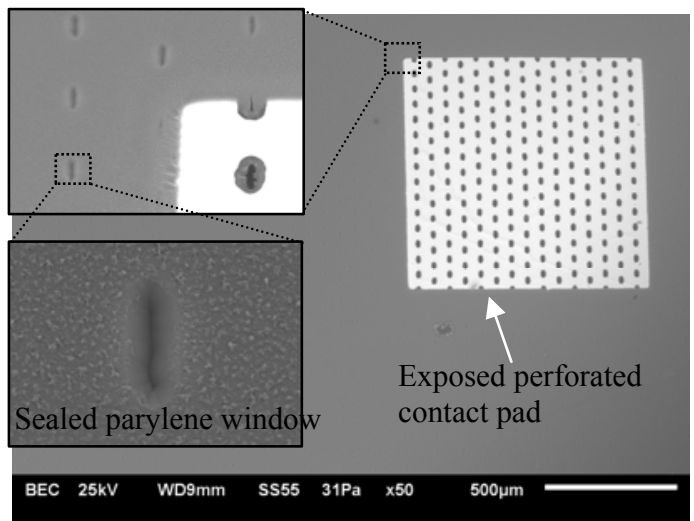

Figure 5: Front side SEM image of the device

Figure 6 shows the cross section of the flexible device. The relative position of the device layer in thickness direction of the parylene $\mathrm{C}$ film can be observed. Note that there was an initial parylene film above the device layer and during the resealing process, there was more parylene deposited on the front side after the windows were sealed. Therefore, the device layer was not positioned at the middle plane of the parylene $\mathrm{C}$ layer. The relative position can be easily adjusted by thinning down either top or bottom side of the parylene $\mathrm{C}$ film via $\mathrm{O}_{2}$ plasma.

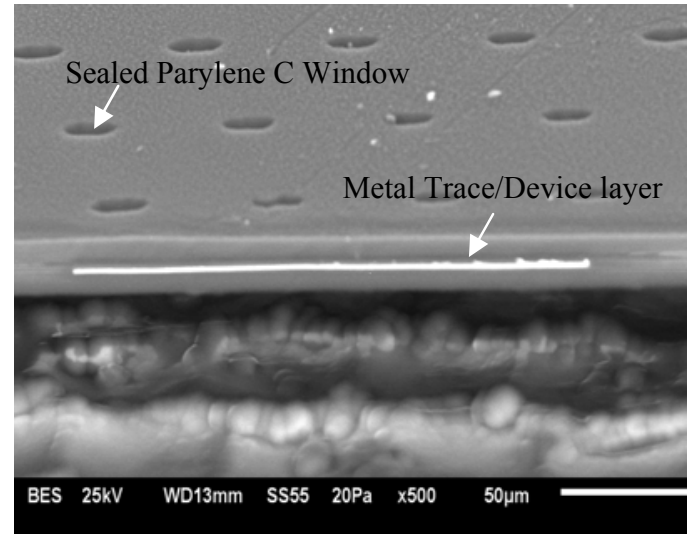

Figure 6: Cross sectional view of one device took by SEM

In order to test the performance of the flexible strain gauge, conductive epoxy was used to connect the testing wires to the exposed $\mathrm{Au}$ contact pads. A simple experiment was carried out by pushing the flexible device in the longitudinal direction (to induce buckling) as illustrated in Fig. 7. The displacement was controlled using a precision micro-manipulator. The resistance change as a function of displacement was recorded. The experiments were repeated 10 times and the averaged result with standard deviation is plotted in Fig. 8. If the gauge factor is 10 (for heavily doped n-type silicon), the strain experienced by the strain gauge is $0.387 \%$ for $1 \mathrm{~mm}$ displacement in the experiment (corresponding to a bending radius of $\sim 3 \mathrm{~mm}$ ). This is actually a sensitive flexible strain gauge because the silicon device layer is not at the neutral plane. It will become even more sensitive if we selectively thin down the bottom side of the parylene $\mathrm{C}$ film and thus move the device layer further away from the neutral plane. For other applications, the device layer can be positioned right on the neutral plane to minimize strains by reducing the thickness of the top parylene $\mathrm{C}$ layer via $\mathrm{O}_{2}$ plasma.

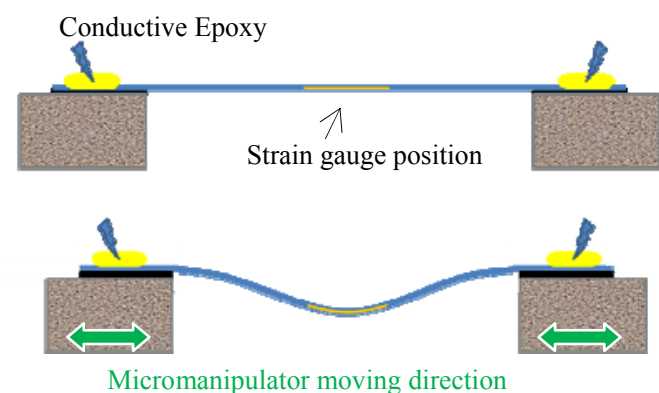

Figure 7: Testing setup with two moving stages to control the displacement.

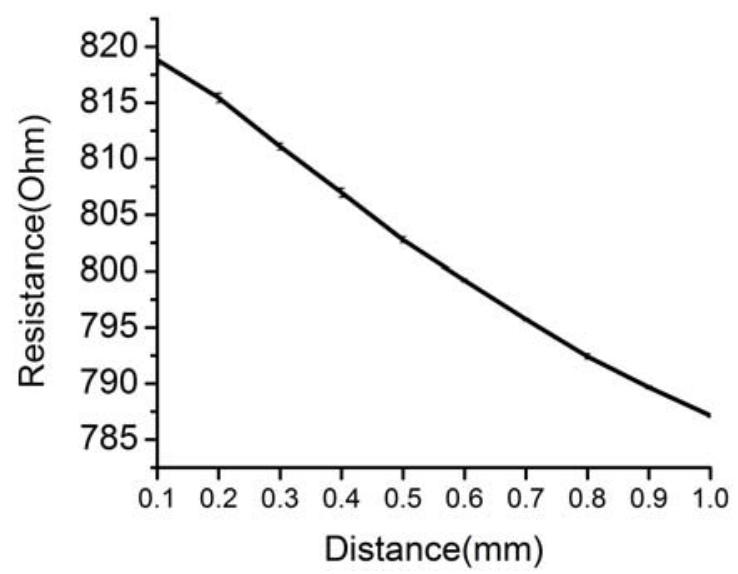

Figure 8: A simple experiment records the resistance change while the device bends when we applied the displacement in the longitudinal direction.

Note for the proof of concept, only simple strain gauges were reported in this paper. We are currently integrating MOSFETs with the flexible substrate to demonstrate more functionalities of our technology.

\section{CONCLUSION}

The biggest advantages of this new flexible electronics technology are its simplicity and compatibility with commercial SOI CMOS process. High-density and high-performance CMOS circuits can be first fabricated using SOI foundry (by slight modifying the layout rules) and readily transferred to flexible substrate. The post-CMOS process only consists of two masks, one is used to form etching holes and the other to open the metal pads and cut the outline. Our proposed fabrication process offers many distinctive benefits for flexible electronics applications. The lamination of the electronics between parylene films offers protection against moistures from the environment. Since there is no transfer printing involved, we can significant increase the device density and improve the yield. Moreover, the electronics or sensors can be positioned at either neutral plane or any other selected planes 
by simply modifying the thickness of the top or bottom parylene $\mathrm{C}$ via $\mathrm{O} 2$ plasma, and therefore allowing more design flexibility.

\section{ACKNOWLEDGMENT}

This material is based upon work partially supported by the National Science Foundation under Grant No. 0747620. Any opinions, findings, and conclusions or recommendations expressed in this material are those of the author(s) and do not necessarily reflect the views of the National Science Foundation. The microfabrication was carried out in the $\mathrm{nFAB}$ cleanroom at Wayne State University and LNF at University of Michigan, Ann Arbor.

\section{REFERENCES}

[1] E. Fortunato, I. Ferreira, F. Giuliani, and R. Martins, "Flexible large area thin film position sensitive detectors," Sensors and Actuators A-Physical, vol. 86, pp. 182-186, 2000.

[2] T. Stieglitz, H. Beutel, and J. U. Meyer, "A flexible, light-weight multichannel sieve electrode with integrated cables for interfacing regenerating peripheral nerves," Sensors and Actuators a-Physical, vol. 60, pp. 240-243, May 1997.

[3] M. Boucinha, P. Brogueira, V. Chu, and J. P. Conde, "Amorphous silicon air-gap resonators on large-area substrates," Applied Physics Letters, vol. 77, pp. 907-909, Aug 72000.

[4] Y. Xu, F. Jiang, S. Newbern, A. Huang, C. M. Ho, and Y. C. Tai, "Flexible shear-stress sensor skin and its application to unmanned aerial vehicles," Sensors and Actuators a-Physical, vol. 105, pp. 321-329, Aug 15 2003.

[5] Y. Xu, Y. C. Tai, A. Huang, and C. M. Ho, "IC-integrated flexible shear-stress sensor skin," Journal of Microelectromechanical Systems, vol. 12, pp. 740-747, Oct 2003.
R. B. Katragadda and X. Yong, "A novel intelligent textile technology based on silicon flexible skins," Sensors and Actuators: A. Physical, vol. 143, pp. 169-74, 2008 .

[7] R. B. Katragadda and Y. Xu, "A novel intelligent textile technology based on silicon flexible skins," Sensors and Actuators a-Physical, vol. 143, pp. 169-174, May 22008.

[8] K. J. Lee, M. J. Motala, M. A. Meitl, W. R. Childs, E. Menard, A. K. Shim, J. A. Rogers, and R. G. Nuzzo, "Large-area, selective transfer of microstructured silicon: A printing-based approach to high-performance thin-film transistors supported on flexible substrates," Advanced Materials, vol. 17, pp. 2332-+, Oct 42005.

[9] E. Menard, K. J. Lee, D. Y. Khang, R. G. Nuzzo, and J. A. Rogers, "A printable form of silicon for high performance thin film transistors on plastic substrates," Applied Physics Letters, vol. 84, pp. 5398-5400, Jun 282004.

[10] D. H. Kim, N. S. Lu, R. Ma, Y. S. Kim, R. H. Kim, S. D. Wang, J. Wu, S. M. Won, H. Tao, A. Islam, K. J. Yu, T. I. Kim, R. Chowdhury, M. Ying, L. Z. Xu, M. Li, H. J. Chung, H. Keum, M. McCormick, P. Liu, Y. W. Zhang, F. G. Omenetto, Y. G. Huang, T. Coleman, and J. A. Rogers, "Epidermal Electronics," Science, vol. 333, pp. 838-843, Aug 122011.

[11] J. Viventi, D.-H. Kim, J. D. Moss, Y.-S. Kim, J. A. Blanco, N. Annetta, A. Hicks, J. Xiao, Y. Huang, D. J. Callans, J. A. Rogers, and B. Litt, "A Conformal, Bio-Interfaced Class of Silicon Electronics for Mapping Cardiac Electrophysiology," Science Translational Medicine, vol. 2, Mar 242010.

[12] J. John, Y. F. Li, J. S. Zhang, J. A. Loeb, and Y. Xu, "Microfabrication of 3D neural probes with combined electrical and chemical interfaces," Journal of Micromechanics and Microengineering, vol. 21, Oct 2011.

[13] Y. Li, H. Tu, R. Iezzi, P. Finlayson, and Y. Xu, "Development of Individually-Addressable Parylene Microtube Arrays," Journal of Microengineering and Micromechanics, vol. 21, 2011. 